\title{
Initial experience of Da Vinci robotic thoracic surgery at the First Affiliated Hospital of Zhejiang University
}

\author{
Zhehao He, Liping Zeng, Chong Zhang, Luming Wang, Zhitian Wang, Azmat Rustam, Chengli Du, \\ Wang Lv, Jian Hu \\ Department of Thoracic Surgery, First Affiliated Hospital, College of Medicine, Zhejiang University, Hangzhou 310003, China \\ Correspondence to: Jian Hu. Department of Thoracic Surgery, First Affiliated Hospital, College of Medicine, Zhejiang University, No. 79, Qingchun \\ Road, Hangzhou 310003, China. Email: dr_hujian@zju.edu.cn.
}

\begin{abstract}
Robot-assisted thoracic surgery (RATS) is a relatively new but rapidly adopted technique, pioneered by the urological and gynecological departments. The primary objective of this study is to present the current status, a series of improvement and innovation of Da Vinci robotic surgery in the Department of Thoracic Surgery at First Affiliated Hospital of Zhejiang University. In addition, we discuss the prospect of robotic surgical technology.
\end{abstract}

Keywords: Robotic-assisted thoracic surgery; initial experience; current status

Received: 20 July 2017; Accepted: 14 September 2017; Published: 28 October 2017.

doi: 10.21037/jovs.2017.09.11

View this article at: http://dx.doi.org/10.21037/jovs.2017.09.11

To achieve minimally invasive cardiothoracic surgery was the original purpose of the Da Vinci surgical system. However, it has been popularly used in urology surgeries, which has made urological condition become the first widely adopted surgeries, especially in radical prostatectomy, partial nephrectomy and pyeloplasty, prompting exponentially increased and worldwide popularity of robotic surgery. Currently, the robotic surgical system has been widely adopted and disseminated in the different fields of minimally invasive surgery, such as neck surgery, thoracic surgery, general surgery and Urology (1-4).

Da Vinci's robotic system has evolved into the fourth generation, and the system consists of three main parts: a surgeon's console, a patient-side cart, a high-performance vision system. The lens of the surgeon's console can be magnified 10-15 times in the field of view, in a $3 \mathrm{D}$ stereoscopic image format (5). The robotic arm, which has seven degrees of free motion, can mimic the action of the human hand and wrist with more flexibility. Instruments aligned with the arms can aid to complete different operations such as rotation, stitching and clipping. The Da Vinci's built-in vibration elimination and action calibration system guarantee precision of the robotic arm in a narrow surgical field (6). With the above characteristics of the Da
Vinci surgical system, comparing with the conventional endoscopy, surgeons can improve the capability of precise hemostasis, reduce the operative risk and the damage to the surrounding normal tissue.

The installation of the Da Vinci surgical system (da Vinci Si) was completed at the First Affiliated Hospital of Zhejiang University on September 11, 2014, and on the first roboticassisted liver surgery of Zhejiang province was performed on that same day, marking the beginning of the robotic surgical era. Urological, General, thoracic, cardiac surgical departments, as well as the gynecological department, were the early adoptors of the new technology, and soon after that was a promotion of the Da Vinci system in use in many surgical cases. According to Intuitive Surgical records, more than 2,400 surgical cases were completed at the First Affiliated Hospital of Zhejiang University using the Da Vinci system. As use cases increased, our center set a world record in 2015 , for single unit use with 836 cases, which was broken by our center by reaching 888 cases in 2016 .

The first robotic thoracic surgery at the First Affiliated Hospital of Zhejiang University was performed on September 12, 2014. To date, July 3, 2017, in less than 3 years, 514 cases of robotic thoracic surgery have been successfully completed, making our center rank third in 
Table 1 Types of robotic thoracic surgery

\begin{tabular}{lcc}
\hline Surgery types & No. of cases & Percentage (\%) \\
\hline Pulmonary resection & 495 & 91.16 \\
Mediastinal mass resection & 37 & 6.81 \\
Esophageal tumor resection & 11 & 2.03 \\
\hline
\end{tabular}

China. Surgical procedures including pulmonary lobectomy, segmentectomy, wedge resection, sleeve resection, radical resection of esophageal carcinoma and mediastinal tumor resection have all been performed under the Da Vinci system by put department.

\section{Indication}

The Da Vinci robotic surgical indications at our center are almost identical to thoracoscopic surgery, which are mainly for peripheral lung lesions and selected cases of central type lung lesions, benign tumors of the esophagus, esophageal carcinoma that invades less than adventitia, mediastinal masses with relatively clear boundary, etc.

\section{Clinical characteristics}

The type of surgery mainly covers the pulmonary, mediastinal and esophageal diseases in three categories, and pulmonary surgery accounted for more than $90 \%$ (Table 1). The percentage of female patients who underwent lung surgery with the system was significantly higher than males, accounting for about $60 \%$. Age was normally distributed with a center range of $50-70$ years, which accounted for $64.96 \%$. Lesions were mainly located in the right upper pulmonary lobe $(35.96 \%)$ and the right lower lobe (21.82\%). The main pathological type was adenocarcinoma (65.25\%), and the rest included pulmonary chronic inflammatory lesions, pulmonary granulomatous inflammation, and intrapulmonary lymph nodes. Pulmonary lobectomy $(44.85 \%)$ and wedge resection $(46.26 \%)$ were the main procedures, with $8.69 \%$ segmentectomy and 1 case of the right upper lobe sleeve resection (Table 2). Segmentectomy was mainly performed on the right upper posterior segment, the proper segment of the upper lobe, the lingular segment and the superior segment of the bilateral lower lobes. Patients who received surgery for mediastinal masses were similar in gender. Patients 50-70 years were predominated $(64.86 \%)$, and the most common lesions located in the anterior mediastinum, thymoma, thymic cyst, mediastinal cyst and neurogenic tumours were the main pathological types (Table 3). The majority of patients with esophageal procedures were more than 60 years on average, with mostly squamous cell carcinoma and 1 case of esophageal neurinoma (Table 4).

\section{Safety}

Of all the 514 patients, only 2 pulmonary surgical cases converted to open surgery because of severe pleural adhesion. Three patients (1 cases of pulmonary disease, 2 cases of mediastinal) received a second surgery due to postoperative hemorrhage. There were no conversations to open surgery due to uncontrolled intraoperative bleeding. Intraoperative pulmonary vascular injury was managed by compression hemostasis or suction or preliminary hemostasis by robotic clamp and further suture.

\section{Improvement and innovation}

With the experience in minimally invasive thoracoscopic surgery at our center, combined with the current trend of enhanced recovery after surgery (ERAS), we are continuously improving in technique and innovation in the Da Vinci robotic thoracic surgery.

\section{Flexible assisted port position}

A total of four ports are often placed utilizing three robotic arms for pulmonary surgery. According to the experience of other centers, the locations of ports for the camera and the other two arms are similar. Namely, the camera port generally is placed in the 7 th or 8 th intercostal anterior axillary line, and the other two arm ports placed $8-10 \mathrm{~cm}$ from the camera port. However, the assisted port position is flexible, according to the different surgical methods.

The "one-way type" approach of Liu et al. (7) was followed when thoracoscopic lobectomy was started at our department. The principle of "one-way type" incision design is to make the stapler roughly perpendicular to the target lobe hilar structure, and horizontal with the mediastinum. This approach facilitates stapler placement to divide bronchus and pulmonary vessels, with appropriate residual bronchial and vascular stumps. The endostapler is placed through the assisted port at the 8th or 9th intercostal posterior axillary line for the resection of the upper and middle lobes, and the assisted 
Table 2 Demographics and clinicopathologic characteristics of robotic pulmonary resection

\begin{tabular}{|c|c|c|}
\hline $\begin{array}{l}\text { Demographics or clinicopathologic } \\
\text { characteristics }\end{array}$ & $\begin{array}{l}\text { No. of patient/ } \\
\text { cases }\end{array}$ & $\begin{array}{l}\text { Percentage } \\
\quad(\%)\end{array}$ \\
\hline \multicolumn{3}{|l|}{ Gender } \\
\hline Male & 184 & 39.32 \\
\hline Female & 284 & 60.68 \\
\hline \multicolumn{3}{|l|}{ Age (years) } \\
\hline$\leq 29$ & 5 & 1.07 \\
\hline $30-39$ & 25 & 5.34 \\
\hline $40-49$ & 84 & 17.95 \\
\hline $50-59$ & 152 & 32.48 \\
\hline $60-69$ & 152 & 32.48 \\
\hline $70-79$ & 45 & 9.62 \\
\hline$\geq 80$ & 5 & 1.07 \\
\hline \multicolumn{3}{|l|}{ Tumor location } \\
\hline Right upper lobe & 178 & 35.96 \\
\hline Right middle lobe & 45 & 9.09 \\
\hline Right lower lobe & 108 & 21.82 \\
\hline Left upper lobe & 80 & 16.16 \\
\hline Left lower lobe & 84 & 16.97 \\
\hline \multicolumn{3}{|l|}{ Resection types } \\
\hline Sleeve resection & 1 & 0.20 \\
\hline Lobectomy & 222 & 44.85 \\
\hline Segmentectomy & 43 & 8.69 \\
\hline Wedge resection & 229 & 46.26 \\
\hline
\end{tabular}

Table 2 (continued)

port placed at the 4th intercostal anterior axillary line for the lower lobes.

Based on our experience with the forementioned technique, we advocate that placement of the assisted port is crucial with adjustments made based on to the pulmonary lesion. In resection of the upper and middle lobes, the assisted port is placed at the 9th/10th intercostal posterior axillary line and at the 4th intercostal anterior axillary line for lower lobectomy (Figure 1). This makes the stapler and bronchus, pulmonary artery and pulmonary vein vertical, reduce vascular tension and improve safety. This experience can also be extended to robotic segmentectomy.
Table 2 (continued)

\begin{tabular}{|c|c|c|}
\hline $\begin{array}{l}\text { Demographics or clinicopathologic } \\
\text { characteristics }\end{array}$ & $\begin{array}{l}\text { No. of patient/ } \\
\text { cases }\end{array}$ & $\begin{array}{l}\text { Percentage } \\
\quad(\%)\end{array}$ \\
\hline \multicolumn{3}{|l|}{ Histology } \\
\hline Adenocarcinoma & 323 & 65.25 \\
\hline Chronic inflammation & 38 & 7.68 \\
\hline $\mathrm{AAH}$ & 36 & 7.27 \\
\hline Granulomatous inflammation & 26 & 5.25 \\
\hline $\begin{array}{l}\text { Intrapulmonary lymph nodes/ } \\
\text { fibrotic nodule }\end{array}$ & 20 & 4.04 \\
\hline Squamous cell carcinoma & 15 & 3.03 \\
\hline Hamartoma & 13 & 2.63 \\
\hline $\begin{array}{l}\text { Metastatic tumor (liver, renal, } \\
\text { colon, etc.) }\end{array}$ & 7 & 1.41 \\
\hline Sclerosing hemangioma & 5 & 1.01 \\
\hline $\begin{array}{l}\text { Mycotic infection (aspergillus, } \\
\text { Cryptococcus) }\end{array}$ & 5 & 1.01 \\
\hline $\begin{array}{l}\text { Lymphatic epithelioma like } \\
\text { carcinoma }\end{array}$ & 2 & 0.40 \\
\hline Large cell carcinoma & 1 & 0.20 \\
\hline Poorly differentiated NSCLC & 1 & 0.20 \\
\hline Pulmonary sequestration & 1 & 0.20 \\
\hline Solitary fibrous tumor & 1 & 0.20 \\
\hline Adenosquamous carcinoma & 1 & 0.20 \\
\hline \multicolumn{3}{|c|}{$\begin{array}{l}\text { AAH, atypical adenomatous hyperplasia; NACLC, non-small cell } \\
\text { lung cancer. }\end{array}$} \\
\hline
\end{tabular}

The Da Vinci surgical system is at a disadvantage when it comes to pulmonary nodule localization by finger palpation due to lack of tactile feedback compared with traditional. Hence, localization of nodules by instrument is not dependable during robotic surgery. The innovative use of intraoperative ultrasound for small pulmonary nodules has been employed at our center (Figures 2,3). The study showed (8), for pulmonary nodules located less than $2 \mathrm{~cm}$ from the visceral pleura, the sensitivity of ultrasound is $100 \%$ and specificity is $80 \%$, with a total accuracy of $93.3 \%$. Intraoperative ultrasound can also help the differential diagnosis of the nature of the pulmonary nodes. 
Table 3 Demographics and clinicopathologic characteristics of robotic mediastinal mass resection

\begin{tabular}{|c|c|c|}
\hline $\begin{array}{l}\text { Demographics or clinicopathologic } \\
\text { characteristics }\end{array}$ & $\begin{array}{l}\text { No. of patient/ } \\
\text { cases }\end{array}$ & $\begin{array}{l}\text { Percentage } \\
\text { (\%) }\end{array}$ \\
\hline \multicolumn{3}{|l|}{ Gender } \\
\hline Male & 18 & 48.65 \\
\hline Female & 19 & 51.35 \\
\hline \multicolumn{3}{|l|}{ Age (years) } \\
\hline$\leq 39$ & 4 & 10.81 \\
\hline $40-49$ & 7 & 18.92 \\
\hline $50-59$ & 11 & 29.73 \\
\hline $60-69$ & 13 & 35.14 \\
\hline$\geq 70$ & 2 & 5.41 \\
\hline \multicolumn{3}{|l|}{ Tumor location } \\
\hline Anterior mediastinum & 30 & 81.08 \\
\hline Middle mediastinum & 1 & 2.70 \\
\hline Posterior mediastinum & 6 & 16.22 \\
\hline \multicolumn{3}{|l|}{ Histology } \\
\hline Thymoma & 11 & 29.73 \\
\hline Thymic hyperplasia & 3 & 8.11 \\
\hline Thymic cyst, mediastinal cyst & 15 & 40.54 \\
\hline Teratoma & 2 & 5.41 \\
\hline $\begin{array}{l}\text { Schwannoma, ganglion cell } \\
\text { neuroma }\end{array}$ & 5 & 13.51 \\
\hline Atypical carcinoid & 1 & 2.70 \\
\hline
\end{tabular}

Table 4 Demographics and clinicopathologic characteristics of robotic esophageal tumor resection

\begin{tabular}{lcc}
\hline $\begin{array}{l}\text { Demographics or } \\
\text { clinicopathologic characteristics }\end{array}$ & $\begin{array}{c}\text { No. of patient/ } \\
\text { cases }\end{array}$ & $\begin{array}{c}\text { Percentage } \\
(\%)\end{array}$ \\
\hline Gender & 7 & 63.64 \\
Male & 4 & 36.36 \\
Female & & \\
Age (years) & 2 & 18.18 \\
$\leq 59$ & 5 & 45.45 \\
$60-69$ & 4 & 36.36 \\
$\geq 70$ & & \\
Histology & 10 & 90.91 \\
Squamous cell carcinoma & 1 & 9.09 \\
Schwannoma & & \\
\hline
\end{tabular}

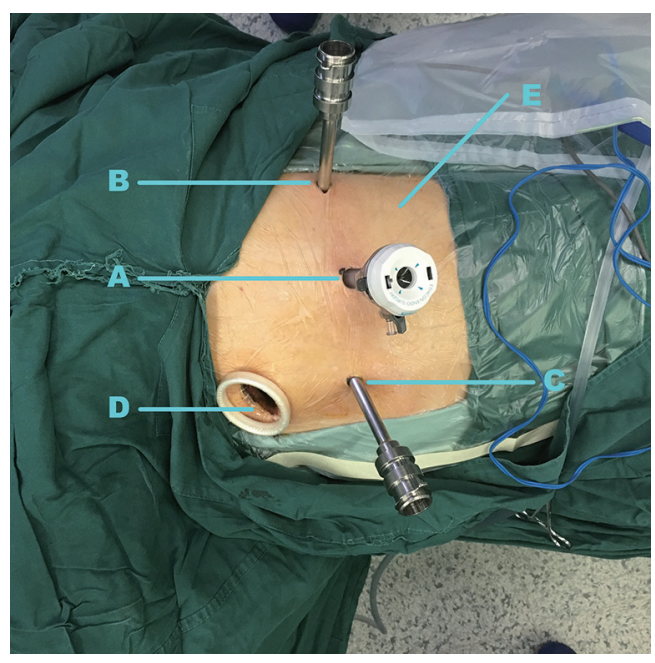

Figure 1 Patient positioning and port placement. (A) Camera port; $(\mathrm{B}, \mathrm{C})$ arm port; (D) the assisted port placed in the 4th intercostal anterior axillary line for lower lobectomy; (E) the assisted port placed in the 10th intercostal posterior axillary line for upper lobectomy.

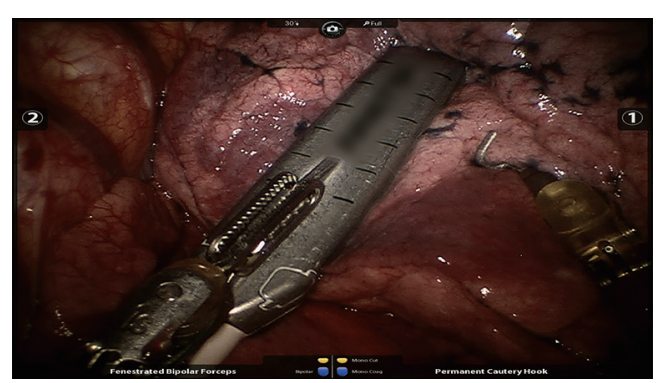

Figure 2 The localization of pulmonary nodes using en ultrasound probe holding by instrument (Fenestrated Bipolar Forceps).

\section{Optimized thoracic drainage strategy}

With the rapid development of minimally-invasive thoracic surgery and the widely accepted concept of enhanced recovery after surgery (ERAS), the problems with routine postoperative thoracic drainage tube have become increasingly prominent, such as the increase of postoperative pain, delayed wound healing, etc. $(9,10)$. Considering that the pleural cavity is a negative pressure closed cavity, the indication of no postoperative chest tube drainage should be rigorously selected. With the Da Vinci system's advanced features, surgical trauma is reduced, and postoperative drainage is not needed in some cases (11), which remarkably increases the feasibility of optimization of the postoperative 


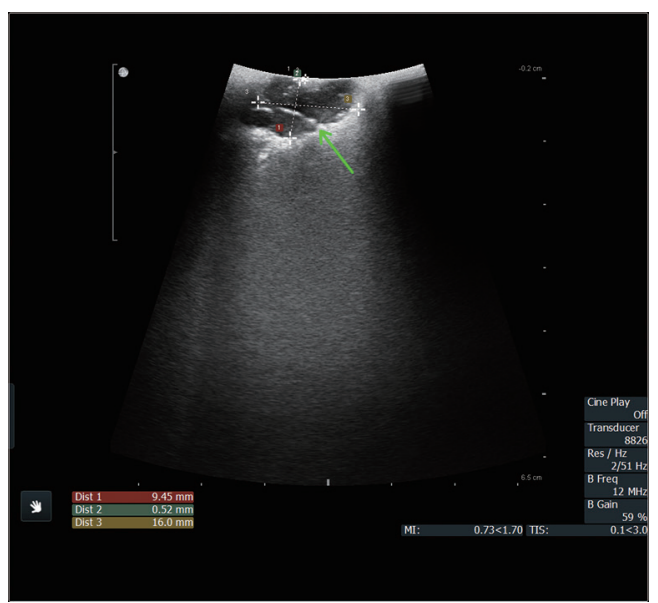

Figure 3 Ultrasound image shows the pulmonary node (green arrow).

drainage strategy. Our center put forward and explored the optimized drainage strategy of robotic thoracic surgery (12), four options would be applied, including no tube placement, deep venous catheter placement connecting to drainage pack, $18 \mathrm{~F} / 30 \mathrm{~F}$ silicone tube connected to a water-sealed bottle. According to the surgical type and in patients with systemic conditions, there are options such as placement of drainage tube or placement of deep vein catheter drainage bag. With the optimized drainage strategy, not only is the drainage effect satisfactory, but also a further decrease in the postoperative pain. Ultimately, this contributes to promoting rapid recovery after surgery for patients.

\section{Learning curve}

In our center, there are in total five surgeons can perform Da Vinci robot thoracic surgery. Evaluation of surgical proficiency level is a difficult process, as calculation of operation time is often subject to surgery, anatomical variation, chest adhesions and other effects. So, we apply self-evaluation interview to evaluate the proficiency for robot chest surgery of each operator.

The interview results showed that 4 out of 5 operators are experienced in thoracoscopic surgery before the Da Vinci robot surgery. In average, the operators can proficiently use the Da Vinci robot surgery system after 7 (range, 5-10) cases, and can master the technique of Da Vinci robot anatomical lung resection after 14 (range, 5-30) cases (Segment resection or lobectomy). All the five operators believe that the previous thoracoscopic surgery experience is identical to learning $\mathrm{Da}$ Vinci robot surgery.
But one of them also think that excessively relying on previous thoracoscopic surgery experience may cause some confusion while learning Da Vinci robot surgery.

In conclusion, Da Vinci robot surgery is easy to learn and master, especially for those who are already experienced in thoracoscopic surgery. In addition, the beginner of Da Vinci robot thoracic surgery work with experienced assistant who sets at the operation table will speed up the learning.

\section{Outlook}

Intuitive Surgical launched the first commercial Da Vinci surgical system in 1996. Recently, Johnson, Google and other well-known companies have invested heavily in the field of surgical system, which has made possible the development of the fourth generation Da Vinci surgical system. Although the Da Vinci surgical system has seen a constant update in the last 20 years, the function and development of the system is still far from reaching the replacing the function of the surgeon.

\section{Diversification of arm selection}

In general, the robotic-assisted port is used for retraction, stapling, suctioning and surgical specimen retrieval. Now, the robotic Stapler is already available on the arm of the fourth generation Da Vinci surgical system (da Vinci Xi) $(13,14)$. Through the flexible rotation of arm, the endostapler direction can be adjusted vertical to the hilar structure, without the need for an assisted port. Through the assisted port, the oval forceps can be used for surgical field exposure. Intuitive Surgical has also launched an arm designed for retraction. With progress like these, the assist port may no longer be needed when a suction arm or robotic instrument with suction function is designed in future updates to the system; This can further contribute to the reduction of surgical trauma and postoperative pain.

\section{Single-port surgical system}

Lens and the arm designed for the single-port surgical system have been available for da Vinci Si, and several studies have reported its application in laparoscopic surgery $(15,16)$. However, the problem of endostapler remained unsolved. Currently, surgery without the use of endostapler can be carried out in cases such as mediastinal tumor resection and esophageal benign tumor resection. 


\section{Miniaturization}

After 20 years of evolution, compared with the first generation of products, the newest surgical system is more slender and sophisticated, but the whole system still occupies a relatively large space. Therefore, the miniaturization of the surgical system would be one of the biggest advancements. An innovative minimized robotic surgical system is developed (17), which can be fixed on the human body and remote controlled. In the near future, we believe that the surgical system would be as small as the conventional surgical instrument, or even completely placed in the body cavity of patients and remotely controlled.

\section{Force feedback}

Force feedback is an important form of feeling in the surgery, second to vision, accessing tissue and disease information. With thoracic surgery, force feedback is needed and essential in localizing small pulmonary nodules, suture, etc. There are some technical options: Firstly, virtual force feedback (18), namely, the resistance information received from the arm appears in the form of color display, sent to the surgeon's screen. Secondly, vibration feedback (19), significantly improved palpation sensation in the robotic surgical arm. Thirdly, the direct force feedback (20), the resistance of the arm is directly sensed by the surgeon's fingers, which can be relatively difficult but most close to the practical surgical condition. The latest da Vinci Xi does not provide the surgeon with force feedback at present, but we believe the forementioned innovative technology is being incorporated into future updates or the next generation products.

\section{Artificial intelligence}

Nowadays, the surgical system has extended the surgeon's hands and eyes, with scarcely any intelligent function. With the great advancement of artificial intelligence in recent years, the future surgical system may possess the functions of surgical decision, dangerous action warning, and possibly neural control.

\section{Economization}

The price of Da Vinci robotic surgical robot system is relatively high, and can only be reused 10 times. Moreover, there are no alternative surgical systems, the cost of robotic surgery is much higher than traditional thoracoscopic surgery and open surgery (21). With the increased competition in the field of surgical robots and the development of domestic surgical system, we hope the cost will greatly decrease and robotic surgery will be further popularized.

\section{Summary}

Since the introduction of the Da Vinci surgical system in 2014, great progress has been made at the department of thoracic surgery of the First Affiliated Hospital of Zhejiang University. The total surgeries performed has exceeded 500 cases, which ranks third in China. Although the expensive cost and other factors limit its wide application in China, with the rapid development of technology and sustaining input of capital, the surgical system will gradually overcome the existing limitations, and will challenge the traditional position of minimally invasive surgical techniques represented by laparoscopy and thoracoscopy, creating a new era of medicine.

\section{Acknowledgements}

Funding: This work was supported by The National Key Research and Development Program of China (No. 2017YFC0113500); The Key Project of Zhejiang Province Science and Technology Plan, China (No. 2014C03032); The Key Project of Zhejiang Province Traditional Chinese Medicine Science and Technology Plan, China (No. 2015ZZ007), and The Foundation of Zhejiang Provincial Traditional Chinese Medicine Scientific Research Plan (No. 2016ZA125).

\section{Footnote}

Conflicts of Interest: The authors have no conflicts of interest to declare.

Informed Consent: Written informed consent was obtained from the patient for publication of this manuscript and any accompanying images.

\section{References}

1. Yu HY, Hevelone ND, Lipsitz SR, et al. Comparative analysis of outcomes and costs following open radical cystectomy versus robot-assisted laparoscopic radical 
cystectomy: results from the US Nationwide Inpatient Sample. Eur Urol 2012;61:1239-44.

2. Pelletier JS, Gill RS, Shi X, et al. Robotic-assisted hepatic resection: a systematic review. Int J Med Robot 2013;9:262-7.

3. Robertson C, Close A, Fraser C, et al. Relative effectiveness of robot-assisted and standard laparoscopic prostatectomy as alternatives to open radical prostatectomy for treatment of localised prostate cancer: a systematic review and mixed treatment comparison meta-analysis. BJU Int 2013;112:798-812.

4. Spinoglio G. editor. Robotic Surgery: Current Applications and New Trends. Springer-Verlag Mailand, 2015:15-176.

5. Byrn JC, Schluender S, Divino CM, et al. Threedimensional imaging improves surgical performance for both novice and experienced operators using the da Vinci Robot System. Am J Surg 2007;193:519-22.

6. Bodner J, Wykypiel H, Greiner A, et al. Early experience with robot-assisted surgery for mediastinal masses. Ann Thorac Surg 2004;78:259-65; discussion 265-6.

7. Liu L, Che G, Pu Q, et al. A new concept of endoscopic lung cancer resection: Single-direction thoracoscopic lobectomy. Surg Oncol 2010;19:e71-7.

8. Zhou Z, Wang Z, Zheng Z, et al. An "alternative finger" in robotic-assisted thoracic surgery: intraoperative ultrasound localization of pulmonary nodules. Med Ultrason 2017. [Epub ahead of print].

9. Mao M, Hughes R, Papadimos TJ, et al. Complications of chest tubes: a focused clinical synopsis. Curr Opin Pulm Med 2015;21:376-86.

10. Cerfolio RJ, Bryant AS. The management of chest tubes after pulmonary resection. Thorac Surg Clin 2010;20:399-405.

11. Zhang D, Xu S, Ding R, et al. A Comparative Study about Trauma after Da Vinci Robotic Surgery and VideoAssisted Mini-Thoractomy. Trauma Crit Care Med 2014;2:296-300.

12. He Z, Yang Y, Zhang C, et al. Optimized Thoracic Drainage Strategy After Da Vinci Robot-assisted

doi: 10.21037/jovs.2017.09.11

Cite this article as: He Z, Zeng L, Zhang C, Wang L, Wang Z, Rustam A, Du C, Lv W, Hu J. Initial experience of Da Vinci robotic thoracic surgery at the First Affiliated Hospital of Zhejiang University. J Vis Surg 2017;3:153.
Thoracic Surgery. Chin J Clin Thorac Cardiovasc Surg 2015;22:919-22.

13. Pearlstein DP. Robotic Lobectomy Utilizing the Robotic Stapler. Ann Thorac Surg 2016;102:e591-3.

14. Holzmacher JL, Luka S, Aziz M, et al. The Use of Robotic and Laparoscopic Surgical Stapling Devices During Minimally Invasive Colon and Rectal Surgery: A Comparison. J Laparoendosc Adv Surg Tech A 2017;27:151-5.

15. Mattevi D, Luciani LG, Vattovani V, et al. First case of robotic laparoendoscopic single-site radical prostatectomy with single-site VesPa platform. J Robot Surg 2017. [Epub ahead of print].

16. Maurice MJ, Kaouk JH. Robotic radical perineal cystectomy and extended pelvic lymphadenectomy: initial investigation using a purpose-built single-port robotic system. BJU Int 2017. [Epub ahead of print].

17. Kawashima K. Developments of surgical assist robot: current and future. Nihon Rinsho 2016;74:109-13.

18. Lécuyer A. Simulating Haptic Feedback Using Vision: a Survey of Research and Applications of "PseudoHaptic Feedback". Presence: Teleoperators and Virtual Environments 2009;18:39-53.

19. Pacchierotti C, Prattichizzo D, Kuchenbecker KJ. Cutaneous Feedback of Fingertip Deformation and Vibration for Palpation in Robotic Surgery. IEEE Trans Biomed Eng 2016;63:278-87.

20. Willaert B. A Mechatronic and Model-Mediated Approach to Bilateral Teleoperation LoTESS: Towards a Force Feedback Telesurgical System (Een mechatronische en model-gebaseerde aanpak van bilaterale teleoperatie LoTESS: een telechirurgisch systeem met krachtterugkoppeling). 2012. Available online: https:// lirias.kuleuven.be/handle/123456789/338080

21. Kajiwara N, Patrick Barron J, Kato Y, et al. CostBenefit Performance of Robotic Surgery Compared with Video-Assisted Thoracoscopic Surgery under the Japanese National Health Insurance System. Ann Thorac Cardiovasc Surg 2015;21:95-101. 